\title{
INFLUENCE OF NITROGEN ON HIGH-FREQUENCY PERMEABILITY OF SOFT MAGNETIC NANOPARTICLES EMBEDDED INTO ALUMINA MATRIX
}

\author{
A. V. LARKIN, Yu. M. ILYASHUK \\ Department of Energy Physics, Belarusian State University \\ Nezavisimosti Ave. 4, 200030 Minsk, Belarus
}

\author{
V. A. KALAEV, Yu. E. KALININ, A. V. SITNIKOV, K. A. SITNIKOV \\ Department of Solid State Physics, Voronezh State Technical University \\ Moskovski Ave. 14, 394026 Voronezh, Russia
}

\begin{abstract}
CoFeZr- $\mathrm{Al}_{2} \mathrm{O}_{3}$ nanocoposite films of $3-5 \mu \mathrm{m}$ thickness, containing metallic alloy nanoparticles embedded into dielectric alumina matrix have been deposited using magnetron sputtering of a composite target in $\mathrm{Ar}$ and $\mathrm{Ar}+\mathrm{N}_{2}$ gas ambient. A behavior of complex high-frequency permeability on content of the metallic phase depends critically on sputtering atmosphere. Nitrogen in the sputtering chamber is shown to retain the super-paramagnetic state of the nanocomposites.
\end{abstract}

\section{Introduction}

FeCo-based ferromagnetic alloy nanoparticles embedded into an amorphous dielectric matrix rank a special place among nanostructured materials. Though looking homogeneous at a macroscopic scale, such binary composite structures are disordered and inhomogeneous at a nanoscale. Depending on the metallic phase concentration $x$, the electrical properties of such mixtures can be varied between the properties of matrix and filler. Such binary composite materials are usually characterized by a critical concentration $x_{\mathrm{C}}$, the percolation threshold [1], when electrically contacting particles of the filler (metallic phase) form a continuous conducting cluster between electric probes of the sample.

Important applications of these systems are conductive adhesives protection layers against electromagnetic radiation and novel electronic and magnetoelectronic devices [2]. Note, that granular metal-dielectric nanocomposites (MDNC) with randomly distributed metallic nanoparticles in a dielectric matrix show extreme characteristics (including the highest tunneling magnetoresistance (TMR) effect) just at approaching the $x_{\mathrm{C}}$ when metallic nanoparticles only begin to form continuous current conducting metallic network. This makes MDNCs with the percolative configuration very attractive for manufacturing of magnetosensors working in high-frequency regimes. 
The goal of this paper is to investigate the influence of the deposition ambient on the high-frequency permeability of the composite films (FeCoZr)$\left(\mathrm{Al}_{2} \mathrm{O}_{3}\right)$ deposited in pure argon or argon-nitrogen gas mixture.

\section{Experimental}

The $\left(\mathrm{Co}_{45} \mathrm{Fe}_{45} \mathrm{Zr}_{10}\right)_{x}\left(\mathrm{Al}_{2} \mathrm{O}_{3}\right)_{100-x}$ films with the fractions of metallic alloy $20<x<65$ at.\% were sputtered in a chamber evacuated and then filled either with pure Ar under pressure $\mathrm{P}_{\mathrm{Ar}}$ of $6.7 \cdot 10^{-2} \mathrm{~Pa}$ (films of set 1) or with $\mathrm{Ar}+\mathrm{N}_{2}$ gas mixture under $\mathrm{P}_{\mathrm{N}}=(1.31-2.13) \cdot 10^{-2} \mathrm{~Pa}$ (set 2).

Complex permeability at $20-200 \mathrm{MHz}$ was measured at room temperature. The real $\left(\mu^{\prime}\right)$ and imaginary $\left(\mu^{\prime \prime}\right)$ parts of the magnetic permeability $\mu$ were recorded by a resonance method [3] at $25-200 \mathrm{MHz}$, with the alternating probing field $B_{\sim}$ oriented in the film plane and parallel to the sample axis. The relative error for the measured $\mu$ was about $8 \%$.

The structure of the as-deposited film composites was investigated using transmission (TEM) and scanning (SEM) electron microscopes. TEM confirmed granular structure of the films with dimensions of metallic nanoparticles of 2-10 nm embedded into amorphous alumina [3-5].

Elemental composition was controlled using a special micro-probe X-ray analyzer in SEM LEO 1455VP.

Preliminary investigation of the Mössbauer spectra, electric and magnetic properties of the MDNCs have revealed that their magnetic state and carrier transport properties were strongly dependent on the metal-to-dielectric ratios $x$ [4,5]. In particular, before the percolation threshold $\left(x<x_{\mathrm{C}} \approx 45-47\right.$ at.\%) nanocomposites of set 1 have shown the superparamagnetic state whereas beyond $x_{\mathrm{C}}$ they were in a ferromagnetic state. Our earlier experiments have shown that for the films of set 2 deposited in the mixture of Ar with reactive gases (oxygen, nitrogen) the range of superparamagnetic state was expanded far beyond $x_{\mathrm{C}}$ observed for the set $1[4,5]$. The latter became apparent in a lack of sextet in Mössbauer spectra [5], conservation of non-hysteretic behavior of magnetization curves higher than the blocking temperature [4,5] and lack of metallic conductance $[4,5]$ far beyond $x_{C}$ in comparison with the films sputtered in pure argon. In accordance with the data of Mössbauer spectroscopy, such peculiarities can be attributed to the formation of oxide or nitride shells around metallic nanoparticles preventing the magnetic interaction between them even at the close electric contact [4]. 


\section{Results and discussion}

The dependence of complex high-frequency permeability on ratio $x$ of the metallic phase is shown in Figs. 1 and 2. Analysis of these figures and earlier results obtained in [3-5] allows us to conclude that the values of complex magnetic permeability and magnetic state of the composites are strongly dependent on sputtering atmosphere and the partial pressure of the reactive gas (nitrogen) in the gas mixture. The maximum for the samples of set 1 just fall at $x_{\mathrm{C}}$ whereas for the set 2 the maximum is shifted to the higher concentrations of the metal fraction. The local maxima of $\mu^{\prime}(x)$ at $42-43$ at.\% in Figs. $2 a$ and $2 b$ may be attributed to the lack of nitride shells on part of metallic nanoparticles.

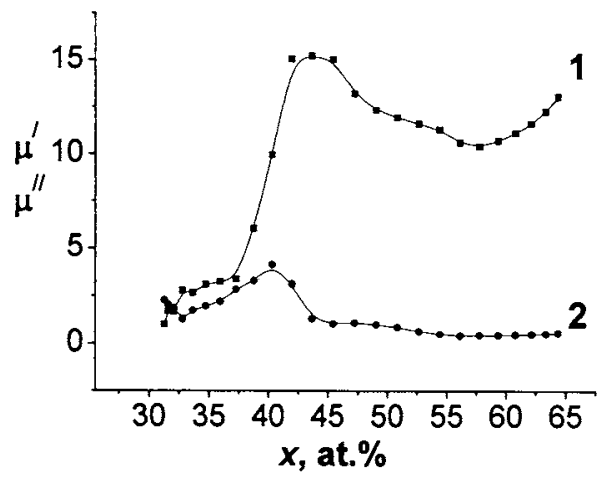

Figure 1. Real $\mu^{\prime}$ (curves 1) and imaginary $\mu^{\prime \prime}$ (curves 2 ) parts of permeability for $f=25 \mathrm{MHz} v s$ concentration $x$ for nanocomposites deposited in Ar.
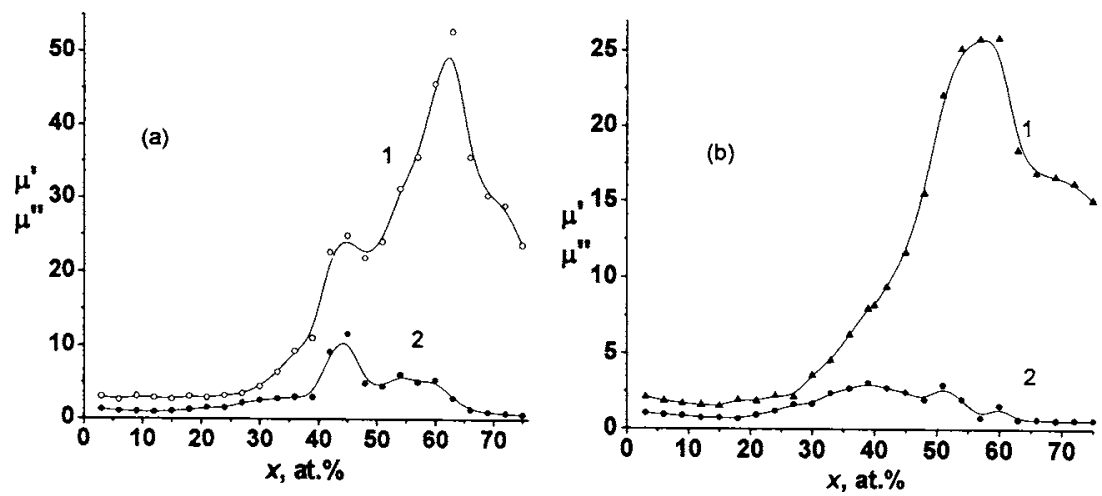

Figure 2. Real $\mu^{\prime}$ (curves 1) and imaginary $\mu^{\prime \prime}$ (curves 2) parts of complex permeability for $f=25 \mathrm{MHz}$ vs metallic-to-dielectric phases ratio $x$ in the nanocomposites of set 2 deposited in argon-nitrogen gas mixture with $P_{N_{2}}=1.31 \cdot 10^{-2} \mathrm{~Pa}$ (a) and $P_{N_{2}}=2.13 \cdot 10^{-2} \mathrm{~Pa}$ (b) 
Thus, three main features are characteristic for the nanocomposites under study.

Firstly, at room temperature the low- $x$ composites display low complex magnetic permeability (compare Figs. 1 and 2 at $x<x_{\mathrm{C}}$ ). These samples also reveal high DC resistance and lack of hysteresis for the magnetization curves and sextet component in the Mössbauer spectra that can evidence a superparamagnetic state.

Secondly, the presence of the first feature far below $x_{C}$ is not generally dependent on conditions of the film preparation including the composition and partial pressure of gaseous atmosphere during film deposition.

Thirdly, the superparamagnetic state of the studied films is shifted to higher $x$ (far beyond the percolation threshold) by addition of nitrogen into the vacuum chamber.

\section{Conclusion}

The behavior of complex high-frequency permeability on the fraction of metallic alloy $x$ for the $\left(\mathrm{Co}_{45} \mathrm{Fe}_{45} \mathrm{Zr}_{10}\right)_{x}\left(\mathrm{Al}_{2} \mathrm{O}_{3}\right)_{100-x}$ films depends critically on sputtering atmosphere and the partial pressure of the reactive gas (nitrogen) in the gas mixture. Addition of nitrogen into the gaseous ambient stabilizes the superparamagnetic state of the nanocomposites even far beyond the percolation threshold observed in the films deposited in pure argon.

\section{Acknowledgments}

This work was supported by VISBY Program of the Swedish Institute, Belarusian Fundamental Research Foundation by Contract F06R-128 and Russian Foundation for Basic Research under the Grant 06-02-81035.

\section{References}

1. G. Grimmet, Percolation (Springer-Verlag, Berlin, 1999).

2. P. Dhagat, S. Prabhakaran, C.R. Sullivan, IEEE Trans. Magn. 40, 2008 (2004).

3. A.V. Sitnikov, Proc. of Intern. Seminar on Magnetic Films, Small Particles and Nanostructures (Astrakhan, Russia, 2003), p. 75.

4. A. Saad, V.A. Kalaev, J.A. Fedotova, K.A. Sitnikov, Yu.E. Kalinin, A.K. Fedotov, I.A. Svito, Rev. Adv. Mater. Science 8, 34 (2004).

5. A.M. Saad et al., Phys. Stat. Solidi (c) 3, 1283 (2006). 